\title{
The Incidence and Determinants of Waterproof Casting in Pediatric Patients
}

\author{
Amanda VanInwegen* and H Robert Tuten \\ Department of Chemistry, Virginia Commonwealth University School of Pharmacy, USA
}

Received: February 12, 2018; Published: February 22, 2018

*Corresponding author: Amanda VanInwegen, BS Chemistry, College of William and Mary, Pharm D Candidate, Virginia Commonwealth University School of Pharmacy, USA, Tel: (804) 543 9540; Email: amvaninwegen@gmail.com

\section{Abstract}

Background: The study was designed to investigate the prevalence and determinants of waterproof casting in children being treated for a fracture at a pediatric orthopedic surgery clinic. Patients whose fractures need immobilization with a cast are given the option to pay for a waterproof liner, which is not covered by insurance carriers. The determining factors in their decision can include sex, age, time of year, and price.

Methods: Subjects were selected for this study if they were placed in a fiberglass cast within a one-year period. The following information was compiled for each patient: sex, age, date of treatment, type of cast, and type of insurance (private, Medicaid, etc.). Also noted was whether or not the patient opted for waterproof casting. All of the patients were immobilized in a cast for approximately the same length of time.

Results: A total of 2407 casts were applied in the one-year period of the study, 960 (39.9\%) of them with a waterproof liner. The prevalence of waterproof cast use was highest in privately insured patients, for children over the age of five, and during the warmer months. The lowest percentage of waterproof casts was among patients insured by Medicaid, the government program for families with limited financial resources.

Conclusion: Waterproof casts are of value to pediatric patients and their parents because they allow children to continue with their normal hygiene regimen and recreational activities, but at a cost that it considered too high for some. Providing the option of a waterproof cast benefits both the practice and the patient.

Level of Evidence: Level I.

\section{Introduction}

The incidence of fractures in children is significant. From birth to age sixteen, the risk of incurring a fracture is $42 \%$ in boys and $27 \%$ in girls [1]. The majority of these fractures can be treated with cast immobilization, while only $20 \%$ require reduction [2]. Traditional casting technique involves the application of stockinette, followed by a layer of cast padding, and then either fiberglass cast tape or plaster of Paris. Although complications are rare with this technique, the traditional casts do impose limitations on this patient population in regards to sports, water activity, and hygiene. Waterproof cast liners allow for swimming and bathing, making hygiene less of an issue and resolving some issues caregivers experience with non-compliant patients getting their traditional casts wet.In our clinics, patients are given the option to pay the additional cost for a waterproof cast. With these casts, a waterproof cast liner is applied directly to the skin first, followed by the fiberglass cast tape. The liner is made of Gore-Tex, a waterproof fabric composed of stretched polytetrafluoroethylene. The material has billions of microscopic pores that do not allow water to pass through, but allow water and perspiration vapor to escape [3].

A waterproof cast is considered a convenience item by insurance companies, so patients must pay out of pocket for the special lining. The cost varies from $\$ 65$ to $\$ 140$ depending on the size of the cast. The benefits of a waterproof cast are subjective to the patient and the patient's parents. For younger children, it may be easier for parents to sponge bathe the child without getting a traditional cast wet, while older children may prefer the convenience and privacy of a waterproof cast in their daily routine. During the summer, parents may choose to pay the extra cost for their child to be able to swim and play sports, especially in extreme heat. Unfortunately, for some parents, the additional cost is too high and might be the main deterring factor in their decision. The purpose of the study is to determine the prevalence and determinants of waterproof casting in pediatric patients in a private practice setting. The hypothesis of the study is that a much smaller percentage of Medicaid insured 
patients will pay for the waterproof cast as compared to privately insured patients.

\section{Materials and Methods}

Subjects were selected for this study if they were casted with a fiberglass cast within a one-year period at a pediatric orthopedic practice. Only patients eighteen years old or younger who were seen by one of the practice's five pediatric providers were included as subjects for this study.Patients who were casted following a surgical procedure, such as a closed reduction, or as a treatment for toe walking were excluded from the study because they were not given the option for a waterproof cast. These patients were identified using billing codes and information in the electronic medical record database.With these criteria, 2407 patients qualified for the study within the one-year period of May 2015 to April 2016. The following information was compiled for each patient: sex, age at time of treatment, month of treatment, type of cast (short arm, long arm, short leg, or long leg), and type of insurance (private, Medicaid, military, or none).

Also noted was whether or not the patient paid for waterproof casting. All of the patients were casted for approximately three weeks.Of the 2407 patients included, 1325 were male and 1082 were female. Of these patients, 503 were between the ages of zero and five and 1904 were six and older. In the one-year period of this study, 1348 of the patients were casted between April and September, while the remaining 1059 were casted between October and March. As for the type of cast, 1146 were short arm, 565 were long arm, 599 were short leg, and 97 were long leg. Considering the type of insurance,1684 were insured privately, 619 were insured by Medicaid, 69 were insured by federal military family insurance, and 35 were not insured at all.The additional patient cost for the waterproof cast is as follows: $\$ 65$ for a short arm cast, $\$ 75$ for a long arm cast, $\$ 95$ for a short leg cast, and \$140 for a long leg cast.

These prices were provided to parents before they made their decision. It is possible that not all patients were offered the option, a detail that is not recorded in the medical records that were used for this study. Worrisome skin conditions, substantial swelling, and unstable fractures can prevent the patient from having a waterproof cast, but these situations are rare.The waterproof liner used in this study is packaged in 5.5-foot-long rolls. The width of the liner is either 2, 3, or 4 inches. A box of twelve rolls purchased from the supplier costs $\$ 135$ for 2 inch rolls, $\$ 165$ for 3 inch rolls, and $\$ 186$ for 4 inch rolls. A combination of liner widths is used during each cast application. The number of rolls used and the size of the rolls used determine the cost to the practice (Table 1).

Table 1: Approximate expenditure for waterproof liner.

\begin{tabular}{|c|c|c|c|c|}
\hline & Short arm & Long arm & Short leg & Long leg \\
\hline $\begin{array}{c}\text { Average } \\
\text { Expense }\end{array}$ & $\$ 24$ & $\$ 31$ & $\$ 44$ & $\$ 48$ \\
\hline
\end{tabular}

\section{Results}

Overall, 960 of the 2407 pediatric patients (39.9\%) opted for a waterproof cast. Of the privately insured patients, $47.9 \%$ opted for a waterproof cast, while only $18.1 \%$ of Medicaid patients paid the extra cost. This difference is statistically significant $(\mathrm{P}<0.001)$ and therefore the data successfully proves the hypothesis. The statistics for military insured patients are similar to privately insured patients, but just slightly lower at $43.5 \%$. Unexpectedly, $33.3 \%$ of patients without any kind of insurance paid for a waterproof cast. However, the data for these two groups is considered insignificant due to the insufficient sample sizes. The remaining data was analyzed with the military insured and uninsured patients excluded.The data was further broken down by sex, age, and season (Table 2). The sex of the patient is not determined to be an important factor in a parent's decision to get a waterproof cast. However, a marginal difference is present across both insurance types. Slightly more males than females paid for waterproof casts $(P=0.110)$, but at the 0.05 critical alpha level this difference is insignificant and most likely due to the inequality of the two sample sizes.

Age seems to have a small factor in the decision to get a waterproof cast. Significantly more parents paid for a waterproof cast for their children over the age of five as compared to children under the age of six $(\mathrm{P}=0.001)$. This proves that waterproof casting may be considered less necessary for younger children who are still being bathed by their parents and who are not swimming or playing sports. This difference is not present or significant; however, in the Medicaid insured patients.As expected, the number of waterproof casts was most variable between seasons. In the summer (defined as April to September), considerably more patients opted for the convenience of a waterproof cast as compared to in the winter (October to March). This distinction was highly significant in the privately insured group $(\mathrm{P}<0.001)$, but less significant for Medicaid patients $(\mathrm{P}=0.011)$, indicating that it may not be as flexible of a decision for these patients.

Table 2: Percentage of patients who paid for a waterproof cast by sex, age, and season.

\begin{tabular}{|c|c|c|c|c|c|c|c|}
\hline Insurance & & & Sex & & Age & Season & \\
\hline & & Male & Female & $0-5$ & $6+$ & Summer & Winter \\
\hline Private & 47.9 & 49.8 & 45.6 & 39.9 & 49.9 & 55.6 & 38.2 \\
\hline Medicaid & 18.1 & 19.5 & 16.1 & 19.1 & 17.8 & 21.4 & 13.6 \\
\hline Total & 39.9 & 41.4 & 38.1 & 33.6 & 41.5 & 46.0 & 32.1 \\
\hline
\end{tabular}

The time of year seems to have the most effect on whether or not a parent chooses a waterproof cast (Table 2). The data was also analyzed by cost, which is determined by the type of cast (Table 3). For all patients, cost seemed to play a significant role in deciding whether or not to pay for a waterproof cast. The most common waterproof casts were short arm casts. Not only are short arm casts the most commonly applied casts overall, but these casts are also the least disruptive to daily life. For this reason, patients are more 
likely to pay for a waterproof cast so they can continue activities. The percentage dips significantly with just the $\$ 10$ difference from short arm to long arm $(\mathrm{P}<0.001)$. This is most likely not due to the Table 3: Percentage of patients who paid for a waterproof cast by cost.

\begin{tabular}{|c|c|c|c|c|c|}
\hline \multirow{2}{*}{ Insurance } & \multicolumn{2}{|c|}{ Short arm } & Long arm & Short leg & Long leg \\
\cline { 2 - 6 } & \multicolumn{2}{|c|}{$\$ \mathbf{6 5}$} & $\mathbf{\$ 7 5}$ & 43.2 & 14.8 \\
\hline Private & 47.9 & 56.8 & 41.1 & 14.5 & 16.7 \\
\hline Medicaid & 18.1 & 21.2 & 16.1 & 35.2 & 12.4 \\
\hline Total & 39.9 & 47.3 & 34.5 & \\
\hline
\end{tabular}

Most pediatric patients with a long arm cast will stop participating in these activities while in the cast and thus would not need to pay for a waterproof lining. On the other hand, the difference between a $\$ 75$ long arm cast and a $\$ 95$ short leg cast is not significant $(\mathrm{P}=0.802)$. This is presumably because leg casts inhibit sports and water activity more than long arm casts, yet are more difficult to keep out of the shower than long arm casts.The most significant difference is from the short leg cast to the $\$ 140$ long leg cast $(\mathrm{P}<0.001)$. Even with privately insured patients, significantly fewer patients elected to pay for the waterproof lining for a long leg cast. For the Medicaid patients, the difference between the percentages is insignificant (Table 3).

\section{Discussion}

Waterproof casts boasta very high satisfaction rate with patients and especially with parents [2]. Unlike traditional casts, they cause little disruption to child and family activities like vacations, sports, and camps. In addition, they allow for improved ease of hygiene and cause less itch, discomfort, and irritation than cotton cast liners [4]. As for the physicians, waterproof liners are preferred because they cause fewer skin problems requiring dermatological treatment [5]. They also benefit the practice because they prevent inadvertent or unexpected appointments for cast changes. When a patient gets a traditional cast wet, the practice must use additional supplies and clinical time to replace the cast. Another major advantage to the practice is the additional source of revenue. By pricing the casts at more than double the cost of the liner, the practice profited approximately $\$ 43,000$ from waterproof casts in the one-year period of this study.

Furthermore, waterproof casts may attract patients to go to a specific practice over one that does not have the option.Based on the results of this study, the amount that a consumer is willing to pay for the convenience of a waterproof cast can be examined. The perceived value of an item for sale is a function of quality and price price, but to the inconvenience of swimming or playing sports with a long arm cast.
[6]. For waterproof casts, the quality is the convenience that the cast provides in terms of activity and hygiene and this convenience is dependent on age and time of year. There is a widely accepted positive correlation between income and luxury purchases, meaning that price can trump quality in these decisions [7]. Parents with more abundant resources find the quality to be worth the price, while others find the price to be too steep. For privately insured patients, the parents' ability to pay the fee is much more flexible and the time of year and the child's age both contribute to their analysis of the value of the cast. Parents with the financial resources to pay for the cast will make the decision based on this perceived value. Alternatively, waterproof casts are generally an unaffordable convenience item for patients who are insured by Medicaid. Often, the extra price is the only factor that prevents these parents from choosing a waterproof cast, overriding the benefits of the cast. However, $18.0 \%$ of these patients still elected for a waterproof cast, indicating the high value of the cast as an option for all parents. B 6(2): 79-83.

2. Shannon EG, DiFazio R, Kasser J, Gerbino P (2005) Waterproof casts for immobilization of children's fractures and sprains. J Pediatr Orthop 25: 56-59.

3. Kruse RW, Fracchia M, Boos M, Guille JT, Bowen JR, et al. (1991) Goretex fabric as a cast underliner in children. J Pediatr Orthop 11: 786-787.

4. Haley CA, DeJong ES, Ward JA, Kragh JF (2006) Waterproof versus cotton cast liners: a randomized, prospective comparison. Am J Orthop 35: 137140 .

5. Selesnick H, Griffiths GA (1997) waterproof cast liner earns high marks. Phys Sportsmed 25: 67-74.

6. Chaudhuri A, Ligas M (2009) Consequences of value in retail markets. J Retail 85(3): 406-419.

7. Jain S, Khan MN, Mishra S (2015) Factors affecting luxury purchase intention: a conceptual framework based on an extension of the theory of planned behavior. SAJM 22(4): 136-163.

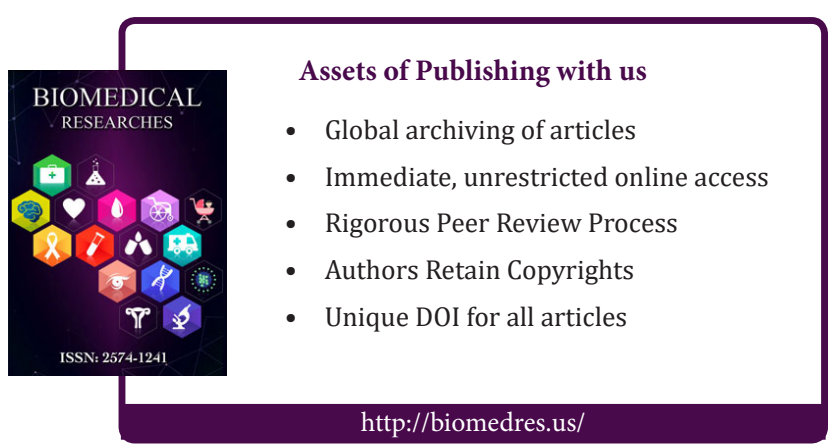

\section{References}

1. Landin L (1997) Epidemiology of children's fractures. J Pediatr Orthop 